\title{
Calidad de vida y educación: mirada contextual de niños, niñas y adolescentes bogotanos
}

\author{
Paola Andrea Riaño Arbeláez ${ }^{* *}$
}

\begin{abstract}
Resumen
Recibido: 12 de febrero de 2014

En este artículo se presentan los resultados de la investigación

Evaluado: 24 de abril de 2014

Prácticas discursivas sobre calidad de vida de niños, niñas y jóvenes en

Aceptado: 10 de mayo de 2014 contextos multidiversos: caso Bogotá. Su objetivo principal fue comprender las prácticas discursivas sobre calidad de vida manifiestas en las narrativas de niños, niñas y adolescentes escolarizados de la localidad de Teusaquillo. Una investigación cualitativa de tipo histórico-hermenéutico, en la cual se utilizaron grupos focales y narrativas, develando principalmente cuatro temáticas: calidad de vida como deseo y expectativa de afecto de los maestros hacia los estudiantes; calidad de vida como educación integral; calidad de vida como la educación soñada, y educación como expectativa potenciadora del proyecto de vida.
\end{abstract}

Palabras clave: calidad de vida, educación, bienestar, felicidad, narrativas, niños y niñas, adolescentes.

\footnotetext{
Este artículo hace parte del proyecto Prácticas discursivas sobre calidad de vida de niños, niñas y jóvenes en contextos multidiversos, producto de la tesis de grado Universidad de Manizales - CINDE. Cómo citar este artículo: Arbeláez Riaño, P. (2014). Calidad de vida y educación: mirada contextual de niños, niñas y adolescentes bogotanos. Hallazgos, 11 (22), pp. 137-158.

"* Profesional en Psicología, Magíster en Educación y Desarrollo Humano del convenio CINDE - Universidad de Manizales (Colombia). E-mail: paokiandrea@gmail.com
} 


\title{
Life and education: Context view it of Bogotá children and adolescents
}

\begin{abstract}
This article presents the results of the research study on Discursive Practices in quality of life in Children and Adolescents from multi-diverse contexts: Bogota Case Study, which goal was to understand the discursive practices in quality of life that manifest themselves in the narratives of schoolchildren, schoolgirls and school adolescents from Teusaquillo area. A qualitative research study from a historical-hermeneutic approach, which used focus groups as well as narratives and got to mainly reveal four themes: quality of life as an affection desire and expectation from teachers toward students, quality of life as comprehensive education, quality of life as a dream education and education as a life project empowering expectation.
\end{abstract}

Keywords: Quality of life, education, welfare, happiness, narratives, children, adolescents. 


\section{Qualidade de vida e educação: visão contextual de meninos, meninas e adolescentes de Bogotá.}

\section{Resumo}

Neste artigo apresentam-se os resultados da investigação Práticas discursivas sobre a qualidade de vida de meninos, meninas e jovens em contextos multidiversos: caso Bogotá. Seu principal objetivo foi compreender as práticas discursivas sobre a qualidade de vida evidentes nas narrativas de meninos, meninas e adolescentes escolarizados da localidade de Teusaquillo. Um estudo qualitativo do tipo histórico hermenêutico, no qual foram usados grupos focais e narrativas, revelando quatro temáticas principalmente: qualidade de vida como desejo e expectativa de carinho por parte dos professores para os alunos; qualidade de vida como uma educação integral; qualidade de vida como a educação sonhada; e educação como expectativa potenciadora do projeto de vida.

Palavras-chave: Qualidade de vida, educação, bem-estar, felicidade, narrativas, meninos e meninas, adolescentes. 


\section{INTRODUCCIÓN}

El término calidad de vida ha sido usado desde las décadas de los sesentas y setentas del siglo anterior como sinónimo de felicidad, bienestar, sentirse bien y tener las necesidades básicas satisfechas; ha sido abordado desde diversas disciplinas como la economía (para medir la satisfacción de necesidades básicas), la psicología (aludiendo al estado de la salud mental), la política (como elemento dentro de las políticas sociales) y la medicina (como indicador de bienestar de los pacientes de cáncer). La expresión ha sido definida por múltiples autores, sin que aún exista un concepto unificado, como lo manifiesta Rueda (2005, citado en Salazar, 2010). El término calidad de vida hace parte de un universo ideológico y no tiene sentido si no está relacionado con un sistema de valores. Esto lleva a pensar en la complejidad del concepto y las diferentes formas de abordarse.

Según la OMS, la calidad de vida es:

La percepción que un individuo tiene de su lugar en la existencia, en el contexto de la cultura y del sistema de valores en los que vive y en relación con sus objetivos, sus expectativas, sus normas, sus inquietudes. Se trata de un concepto muy amplio que está influido de modo complejo por la salud física del sujeto, su estado psicológico, su nivel de independencia, sus relaciones sociales, así como su relación con los elementos esenciales de su entorno. (citado en Salazar, 2010, p. 42)

Tonon (2005) la define como una forma de contribuir al estudio del bienestar de las personas, desde perspectivas tales como el bienestar físico y psicológico, relacionando las necesidades materiales con las socioafectivas, e integrando mediciones psicológicas y psicosociales de percepción y evaluación de las propias experiencias de los sujetos. La noción de calidad de vida, desde la perspectiva de Sen y Nussbaum (2000, citados en Salazar, 2010) precisan lo que ha significado el bienestar y "estar bien": ambos se refieren al bienestar (bienestar y pasarla bien o "estar bien" respectivamente), el primero supone un estado que no es elegido por el sujeto, y el segundo permite aludir a que es la persona quien elige.

Desde la psicología se menciona la definición general de calidad de vida para adultos, como la percepción subjetiva de los efectos de su estado de salud, incluyendo las enfermedades fisiológicas, alteraciones comportamentales y de funcionamiento social; definición que también es aplicable a los niños, las niñas y los adolescentes (NNA), quienes son el foco de interés de la presente investigación, agregando múltiples variables dentro del contexto social en el que se desarrollan: grupo de pares, familia, y entorno escolar (Ravens-Sieberer et al., 2006, citados en J. Castillo, C. Castillo \& Esguerra, 2011).

Eduardo Lora (2008), asesor principal del Departamento de Investigación del Banco Interamericano de Desarrollo, plantea que el concepto calidad de vida necesita ser abordado desde: a) condiciones de vida, b) capacidades, 3) apreciación de la vida y felicidad; reconociendo en este último las limitantes de las fuentes de información subjetiva. Lora plantea que las percepciones sobre calidad de vida responden a patrones psicológicos y culturales, los cuales han sido estudiados a través del uso de encuestas de opinión para 
conocer las percepciones sobre bienestar $y$, a la vez, sobre cómo los individuos evalúan distintos aspectos de su vida, y cuál es el nivel de satisfacción con sus condiciones. En los resultados se encuentran numerosos indicadores de calidad de vida, basados en percepciones que han generado confusión, hay incongruencias entre las percepciones y los indicadores económicos y sociales, que la sociedad ha escogido como objetivos.

La discusión sobre las múltiples definiciones del concepto de calidad de vida refleja la ambigüedad del término y la necesidad de integrar miradas objetivas y subjetivas, que permitan comprender qué es lo que realmente le importa a la gente, pues esto podría ayudar a los gobiernos a priorizar políticas, revelando la necesidad de combinar las dimensiones que forman parte de éste.

El plan de desarrollo 2010-2014 Prosperidad para todos (Departamento Nacional de Planeación [DNP], 2010) para Colombia, traza como objetivo del sistema de protección social mejorar la calidad de vida de los ciudadanos, e incluye no sólo los ingresos, la salud o el acceso a la educación, sino también elementos críticos como la cultura, el deporte o la oferta de actividades de recreación, cuyos efectos repercutirán en las demás dimensiones.

En el mismo documento se dice que la zona Central genera el $73 \%$ del PIB del país, y su ingreso per cápita es entre dos y tres veces el de las demás zonas, excepto la Nororiental, generando grandes disparidades entre las regiones. A nivel social presenta los menores niveles relativos de pobreza, sin embargo la cantidad de población pobre que requiere atención es la más alta del país, por ser la mayor receptora de migrantes por desplazamiento forzado, generando una elevada demanda bienes, servicios públicos $\mathrm{y}$ empleo. Esto mismo reportó el informe Bogotá una apuesta por Colombia, presentado por el PNUD en 2008, en él se plantea que el principal reto es lograr el desarrollo humano para la ciudad y las poblaciones aledañas, puesto que el crecimiento dispar impide la sostenibilidad de la capital, lo que a largo plazo va en detrimento de la calidad de vida y hace que los cambios en el desarrollo social no puedan ser duraderos.

De la misma manera, el artículo 17 de la ley 1098 de Infancia y Adolescencia, se refiere al derecho a la vida, a la calidad de vida y a un ambiente sano; siendo esencial para su desarrollo integral, por lo que se deben generar condiciones que desde la concepción les aseguren cuidado, protección, alimentación nutritiva y equilibrada, acceso a la salud y educación, vestuario adecuado, recreación, vivienda segura y dotada de servicios públicos en un ambiente sano.

El plan de desarrollo "Bogotá positiva: para vivir mejor" contemplaba como uno de sus principios, de política pública y acción, la calidad de vida; el gobierno distrital se comprometió a lograr el mejoramiento de las condiciones de vida, buscando equilibrar el aumento de la población, los recursos disponibles y la protección del medio ambiente, enmarcado en la dinámica de los procesos de la urbanización y del progreso tecnológico (Concejo de Bogotá, 2008).

El Programa Bogotá Cómo Vamos [BCV] (Casa Editorial El Tiempo, Fundación Corona y Cámara de Comercio de Bogotá, 2011) realizó en 2011 una encuesta de percepción de la ciudad 
entre los bogotanos, encontrando que el $56 \%$ se sentía satisfecho con Bogotá como una ciudad para vivir, porcentaje que ha caído notablemente en los últimos 4 años (en 2008 este porcentaje era del $72 \%$ ), lo cual muestra una reacción de los ciudadanos frente a los grandes problemas que vive actualmente Bogotá, y la catastrófica administración del destituido alcalde Samuel Moreno Rojas.

El informe de auditoría gubernamental con enfoque integral (Contraloría de Bogotá, 2010) encontró que las alcaldías locales prácticamente se limitaron a copiar el plan de desarrollo distrital, sin hacer ningún tipo de análisis o adecuación contextual, que es lo que debe encontrarse en esto tipo de planes, para así dar respuesta a las necesidades puntuales de cada uno de los sectores de la ciudad, maximizando los recursos dispuestos para tal fin.

En la localidad de Teusaquillo, donde se desarrolló esta investigación, el Fondo de Desarrollo Local (Contraloría de Bogotá, 2010) calificó como deficiente la gestión de 2009, en lo relacionado con la ejecución de los proyectos de inversión, en la cual se comprometieron los recursos destinados a los proyectos, pero no se ejecutaron en forma oportuna para beneficiar a la población vulnerable del sector. Las cifras muestran la deficiente gestión de la alcaldía local, que contribuye al detrimento de la calidad de vida de los habitantes, pues a pesar de existir los recursos y los programas a nivel distrital, el gobierno local no se preocupa por usar la información existente sobre las características particulares de Teusaquillo y crear sus propios proyectos, o si se crean no se ejecutan, perdiéndose los rubros asignados y toda la planeación, con las implicaciones que esto conlleva.
De otro lado, las cifras del objetivo de participación, contemplado en el plan de desarrollo local, tienen un porcentaje presupuestal de los más bajos, $2.53 \%$, con respecto a otros objetivos, y también es bajo el interés de la comunidad por hacer parte de los proyectos locales, haciendo que no existan programas que permitan un mejor control social sobre los presupuestos locales. Es por ello que el papel de la educación no puede limitarse únicamente a lo académico, sino que la educación inicial, básica, media y superior de calidad, deben contribuir a la formación de ciudadanos éticos, como lo menciona el Plan de Desarrollo Nacional:

Una educación de calidad requiere entonces formar ciudadanos con valores éticos, respetuosos de lo público, que ejercen los derechos humanos, cumplen sus deberes sociales y conviven en paz, e implica ofrecer una educación que genera oportunidades legítimas de progreso y prosperidad, que sea competitiva, que contribuya a cerrar las brechas de inequidad, centrada en la institución educativa, que permita y comprometa la participación de toda la sociedad en un contexto diverso, multiétnico y pluricultural. En síntesis, nos compromete una educación de calidad como el camino a la prosperidad (DNP, 2010, p. 265).

Retomando diferentes aspectos mencionados en este documento, se observa que aunque el gobierno nacional y la ciudad capital, a través del gobierno distrital, ha hecho grandes esfuerzos por mejorar la calidad de vida y el índice de desarrollo humano para sus habitantes, todavía quedan muchas metas por cumplir dentro del plan de desarrollo; además se deben repensar y reestructurar estrategias en orden a obtener cambios realmente significativos, que 
sean percibidos por los ciudadanos. Es importante centrarse en los gobiernos locales, pues son los que pueden contribuir eficazmente al aumento de la participación ciudadana, en procura de proyectos más acordes a las necesidades de los ciudadanos.

A partir de lo anterior, e intentando tener un acercamiento y una mirada diferente a la calidad de vida con relación a los NNA, desde sus propias voces como protagonistas no sólo de las políticas públicas de niñez y adolescencia sino también de los contextos escolares, surge la pregunta: ¿cuáles son las prácticas discursivas sobre calidad de vida, que se manifiestan en las narrativas de NNA escolarizados de la localidad de Teusaquillo en Bogotá?

Para responder a esta pregunta se plantearon tres objetivos específicos, por medio de los cuales se busca describir las prácticas discursivas de calidad de vida, con el fin de comprenderlas y, finalmente, develar el sentido que tienen para un grupo de NNA escolarizados de la capital. Con esta respuesta se espera contribuir a la ampliación y dinamización del concepto de calidad de vida, que en la construcción de indicadores a través de encuestas realizadas a los jefes de hogar, pues son ellos quienes, con criterios preestablecidos por las Instituciones, califican como buena o mala la calidad de vida de los NNA, sin tomar en cuenta su subjetividad y percepción de los contextos particulares en los que se desenvuelven, principalmente el educativo.

\section{Metodología}

En cuanto investigación de tipo cualitativo, se pretende una aproximación global a las situaciones sociales con el fin de explorarlas, describirlas y comprenderlas a partir de los conocimientos que tienen las diferentes personas involucradas en ellas, y no con base en hipótesis externas (Bonilla y Rodríguez, 1997).

La postura epistemológica desde la cual se llevó a cabo este estudio, es de tipo histórico-hermenéutica y, con base en ella, se empleó el método narrativo. Una característica esencial del enfoque hermenéutico, es el múltiple significado que posea el texto que se vaya a interpretar, traspasando el sentido superficial para llegar al sentido auténtico que le dio el autor; esto se logra por medio de la interpretación, que comienza como hipótesis para transformarse en tesis (Beuchot, 2002). Esta postura permitió el acercamiento a las prácticas y discursos de los NNA, y actores institucionales, sobre calidad de vida.

Bruner (1997) plantea la narrativa como un eje de interpretación en la formación subjetiva de la realidad; mediante las narrativas se puede comprender la influencia del mundo sobre el sentido y significado personal. Las construcciones narrativas dan forma a la realidad que crean. La experiencia de cada sujeto sobre los asuntos humanos, como la política, toma la forma de las narraciones que se emplean para contar cosas sobre ella.

Los discursos, tanto los ajenos como los relativos al individuo, son múltiples, diferentes y todos válidos, ya que parten de puntos de vista, prácticas e historias distintas. Por consiguiente, la elaboración del sentido es, para Bruner (1991), "un proceso social, es una actividad que se da dentro de un contexto cultural e histórico". Es así como la cultura y el lenguaje adquieren un papel básico en 
la construcción de significados. Desde esta perspectiva es posible estudiar y comprender cómo la gente organiza su experiencia, sus conocimientos y transacciones relativas al mundo social, y es a esto a lo que buscó acercarse esta investigación, conociendo las prácticas discursivas que NNA han construido sobre la calidad de vida.

También se retomó, para esta investigación, el concepto de narrativa, elaborado por Arenas, Jaramillo y Riaño (2004) a partir de las concepciones de Bruner y Sluzki:

Una construcción que lleva el hilo conductor entre personajes, guion y escenarios, que dan cuenta de los acontecimientos en la vida de los actores, los cuales generan unos significados (a su vez mediados por el lenguaje) que muestran su forma de puntuar la realidad (Jaramillo y Riaño, 2004, p. 46).

Con lo anterior, se invita a pensar la narrativa de una forma dinámica, en la cual los aconteceres de los protagonistas la enriquecen continuamente, dándole la posibilidad de resignificar su trama. Mediante los relatos orales y escritos, expresados en las narrativas, se busca desarrollar, activamente, las percepciones que tienen los protagonistas sobre la categoría calidad de vida, en aras de enriquecerla y resignificarla desde un lenguaje y una forma de vida cotidianas, que amplíen las concepciones ya analizadas. Para lograr todo esto, en el trabajo de campo se propusieron los siguientes momentos:

\section{Primer momento}

Abre con la elección de los participantes, basándose principalmente en el criterio de la disponibilidad y el consentimiento informado de los padres, por su condición de menores de 18 años, así como la firma aceptando su participación en la investigación.

\section{Protagonistas}

Cuatro NNA entre 12 y 16 años de estrato 4, residentes en la localidad de Teusaquillo de la ciudad de Bogotá, estudiantes de diferentes colegios privados bilingües y matriculados en Kumon Instituto de EducaciónUnidad Pablo VI ${ }^{1}$. Esta es una institución privada extraescolar, que brinda a través del estudio de las matemáticas y la lengua materna, la posibilidad de desarrollar potencialidades en cálculo mental, raciocinio lógico, hábito y comprensión de lectura, entre otras capacidades.

De otro lado, se trabajó con dos actores institucionales, el asesor de la Gerencia de Kumon Colombia y la orientadora de la unidad donde asisten los 4 estudiantes participantes en la investigación.

Se conversó con los protagonistas individualmente para escuchar sus narrativas, a través de una entrevista semiestructurada, con la cual se buscó favorecer "el carácter extraordinario de las descripciones coloquiales, poéticas o pintorescas frente a las descripciones técnicas, y la conversación se hace más exploratoria y menos dirigida a un propósito determinado" (White \& Epston, 1993, p. 93).

\footnotetext{
1 Para más información sobre el método Kumon Instituto de Educación puede consultarse la página web www.kumonla.com
} 
No obstante, se quiso evitar la limitación de los participantes, permitiéndoles que se expresaran de la misma forma en que lo hacen en su vida cotidiana. Erlandson afirma que este tipo de entrevista es guiada por un conjunto de preguntas y cuestiones básicas a explorar, pero ni la redacción es exacta ni el orden de las preguntas está predeterminado (citado en Valles, 1999, p. 179); la investigadora pudo construir una guía de tópicos de acuerdo a cada uno de los aspectos en los que deseaba ahondar: cómo conceptuaban calidad de vida, su propia percepción de ser NNA, la relación que encontraban entre la calidad de vida y la educación, etc.

Posteriormente, se trabajó un grupo focal, tipo taller, con los NNA, entendido como "una reunión con modalidad de entrevista grupal abierta y estructurada, en donde se procura que un grupo de individuos seleccionados por los investigadores discutan y elaboren, desde la experiencia personal, una temática o hecho social que es objeto de investigación" (Hospital Pablo VI Bosa, s.f.). En este espacio se retomaron los elementos comunes encontrados en las narrativas, $\mathrm{y}$, a partir de ellos se asignó la tarea de construir un instrumento para medir la calidad de vida, en donde el grupo debía definir los criterios para hacer esta medición.

Todas estas técnicas, al ser cualitativas, sin duda se centran en el sujeto investigador, por lo que no existe un manual que muestre el procedimiento paso a paso para realizarlas (Binimelis \& Canales, 1994), pero es importante mostrar cómo las comprende el equipo de investigación dentro de este estudio. Por ello, el trabajo con las entrevistas y el grupo focal permitió la identificación de categorías que permitieron el análisis de las narrativas: ideas sobre calidad de vida, relación Kumon - calidad de vida, historia en Kumon, idea sobre ser NNA, ventajas y desventajas sobre ser NNA en la casa, el colegio y Kumon, relación entre calidad de vida y calidad de educación, y percepción de su calidad de vida - .

\section{Segundo momento}

Cohen y Manion (1980, citado por Pourtois \& Desmet, 1992, p. 61) definen la triangulación como "el uso de dos o más métodos en la recogida de datos a propósito del estudio de un fragmento de la conducta humana", con ella se busca que, una vez recogida la información a través de las entrevistas semiestructúrales y el grupo focal, se realice una primera interpretación que alimente la categoría inductiva de análisis: concepto de calidad de vida, y, a la vez, dé origen a dos nuevas categorías deductivas: relación entre calidad de vida y educación, y concepto sobre niño, niña y adolescente, que, como el nombre lo indica, nacieron a partir de las discusiones generadas con los participantes (ver gráfico 1). En este artículo se desarrollará la categoría relación entre calidad de vida y educación.

\section{Tercer momento}

Se realizó un segundo proceso de triangulación de la información, en el cual se retomó lo común a ambos instrumentos (grupo focal y entrevistas), a la luz de la teoría con la cual se analizó la información y la construcción de sentido que da el investigador, lo que alimentó nuevamente las categorías deductivas e inductivas de análisis (ver gráfico 2). 
Gráfico 1. Triangulación de instrumentos

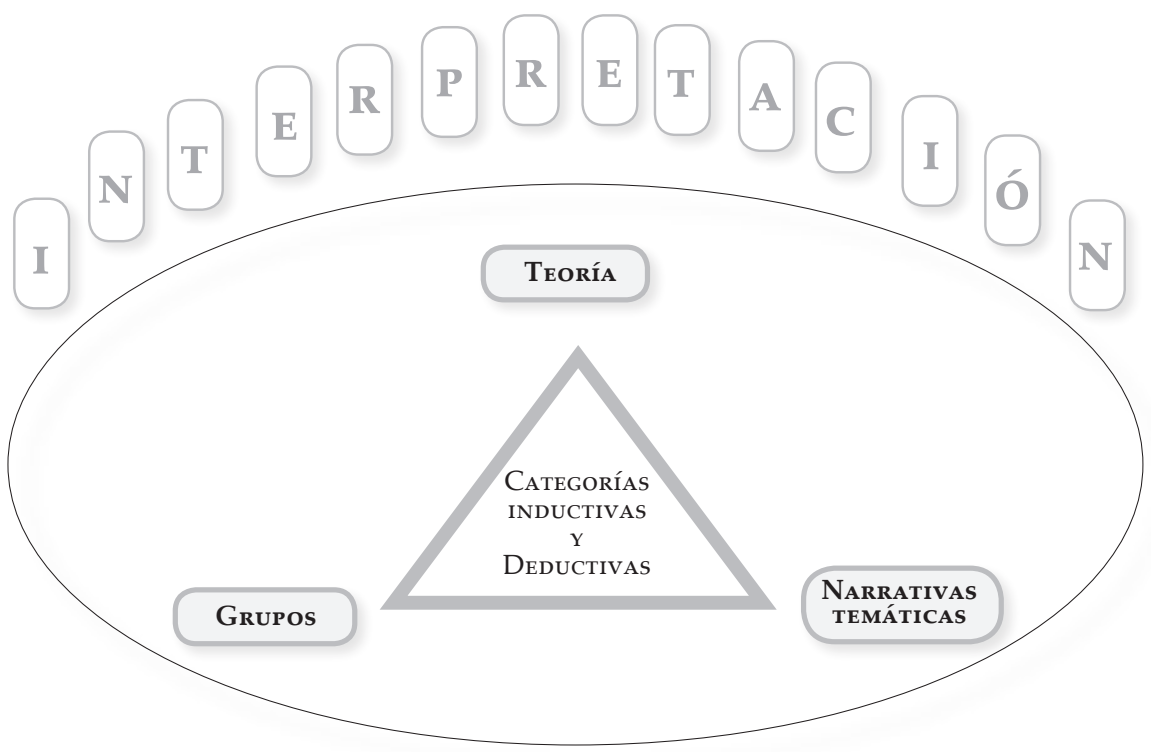

Fuente: Buitrago et al., 2011, p. 54

Gráfico 2. Triangulación interpretativa

INTERPRETACIÓN Y CONSTRUCCIÓN DE SENTIDO

TeORíA

Actores

Fuente: Buitrago et al., 2011, p. 54

Al realizar dos triangulaciones, se buscó validar la información en dos momentos diferentes, a la vez que se alimentaron y cualificaron las categorías de análisis inductivas y deductivas, logrando así un mejor acercamiento a las prácticas y discursos de los participantes en torno a la categoría de análisis.

\section{Relación CALIDAD DE VIDA Y EDUCACIÓN}

Nació como una categoría deductiva de las narrativas de los actores institucionales y los NNA, quienes al tener en común la pertenencia a contextos educativos, se remitían 
constantemente a la educación como elemento importante en su calidad de vida. De sus relatos se derivaron las siguientes subcategorías: 1) calidad de vida como deseo, y expectativa de afecto de los maestros hacia los NNA; 2) calidad de vida como educación integral: relaciones de respeto, reconocimiento y convivencia entre pares y maestros; 3 ) la educación como expectativa potenciadora del proyecto de vida, y 4) calidad de vida como la educación soñada.

\section{Calidad de vida como deseo, y expectativa de afecto de los maestros hacia los NNA}

Esta subcategoría muestra una visión de la calidad de vida como un elemento fundamental dentro del proceso educativo, y ligado al afecto de los maestros hacia los estudiantes, aspecto que se evidenció principalmente en la narrativa de la orientadora, quien cumple la función de guía del proceso dentro de la Unidad de Kumon: Yo lo que siempre he querido con los niños es que ellos sean felices (...) que al mismo tiempo están aprendiendo entonces yo quiero que esa experiencia que ellos están viviendo con el aprendizaje sea una experiencia feliz, que llegar a mi clase de Kumon sea iqué alegría! Su preocupación es por la persona y el aprendizaje para la vida, en la medida en que el estudiante esté bien podrá aprender.

Ya desde Aristóteles (1873), quien no habla de calidad de vida, pero usa los conceptos de felicidad y de buena vida como ejes fundamentales del desarrollo humano, se encuentran elementos que remiten al discurso de esta orientadora, quien pone la felicidad como fin último y necesario para lograr la calidad de vida. Es un estado emocional que remite a la felicidad y que se relaciona nuevamente con la satisfacción personal: para mí la calidad de vida es como la alegría que uno tiene de vivir, que uno sea feliz; la felicidad se puede entender como bienestar. "Se trata de un bien y con frecuencia de una finalidad" (Ferrater, p. 1153, citado en Salazar, 2010, p. 41). Más que relacionar el método Kumon con la calidad de vida, lo vincula con su labor como docente, con el hacer, siendo coherente con su discurso de calidad de vida basado en el ser, hacer y estar. Para Sen (1989, citado en Salazar, 2010):

[...] Si la vida se percibe como un con-
junto de 'haceres y seres' (es decir, de
realizaciones) que es valorado por sí
mismo, el ejercicio dirigido a determi-
nar la calidad de la vida adopta la for-
ma de evaluar estas 'realizaciones' y la
'capacidad para funcionar. (p. 47 )

Lo anterior se relaciona con el desarrollo de la autoestima, que es uno de los objetivos del método Kumon y uno de los procesos involucrados en la generación del bienestar social, que a su vez hace parte de la calidad de vida: el ser, estar y el hacer (Cummins, 1998 citado en Tonon, 2005), como lo menciona la orientadora en otra de sus narrativas: cuando tú te hallas bien, en donde estés, lo que tú hagas, sientas que lo haces bien y además de que lo haces bien, es algo que te gusta hacerlo, un accionar ético y de compromiso con el otro.

Los contenidos, en este caso la matemática y la lengua materna, son un medio para llegar a un fin: la felicidad, "se ha dicho que educar es enseñar a vivir, entendiendo que el fin de tal aprendizaje es que la persona aprenda a ser autónoma y a orientarse de acuerdo con unos principios o valores 
éticos fundamentales" (Camps, 2002). Este proyecto educativo ve al maestro holísticamente, su labor no es sólo la enseñanza de contenidos académicos, sino que involucra también el bienestar de los estudiantes, el cual es visto subjetivamente, por ello la necesidad de preguntarles cómo se sienten. Tonucci (2008) mencionó en una entrevista, que para él "cada momento educativo se debería empezar oyendo a los alumnos", conectándose con la forma de pensar de esta orientadora: Cuando ellos aprenden yo me siento tan contenta o cuando me vienen a contar que me pasó esto en la casa, en el colegio y me cuentan sus cosas yo me siento muy contenta porque sé que si estoy consiguiendo lo que yo más quiero: que no solamente que aprendan sino que ellos tengan una persona con la que puedan hablar, con la que puedan desahogarse, que puedan contar como si fuera una mamá. Su discurso se centra en conectarse con los estudiantes, que son para ella el eje central del proceso, se ve como guía y acompañante del aprendizaje y por ello enfatiza en su relación con los estudiantes.

Renzi y Tonucci (s.f) piensan que hay que resaltar el papel del maestro y considerarlo una pieza esencial en la educación de los niños, ya que algunos niños pasan más tiempo con los maestros que con sus propios padres a lo largo del día, lo que se evidencia en las palabras de esta educadora, que están cargadas de emociones y sentimientos, pues la educación ha hecho parte de su proyecto de vida desde el inicio. Si bien Kumon llega a su vida sin estarlo buscando, encuentra a través de este trabajo "la posibilidad de dar lo mejor de sí, la presencia de alguien en el trabajo que estimule el perfeccionamiento del trabajador, y el hecho de que sus opiniones sean tenidas en cuenta" (Pagés \&
Madrigal, 2008, citado en Matijasevic, Ramírez $\mathcal{E}$ Villada, 2010); todo lo cual se lo permite el ser propietaria de una franquicia, en donde a pesar de seguir unos parámetros generales, tiene un alto de grado de autonomía en las relaciones que establece con los estudiantes y padres de familia, para ella parte esencial del proceso.

\section{Calidad de vida como educación integral: relaciones de respeto, reconocimiento y convivencia entre pares y maestros}

En esta subcategoría, se identificaron discursos que muestran la calidad de vida desde las relaciones entre pares que se tejen en los procesos educativos, aclarando que la mayor parte de los discursos toman como referencia la escuela y Kumon, espacios en los que este grupo pasa la mayor parte del día en el colegio (los horarios de clases oscilan entre las 8 a.m. y 4 p.m., y asisten a Kumon dos veces por semana una hora cada vez). En sus discursos, incorporan la importancia que para ellos tienen elementos como la alimentación escolar, las tareas, los espacios físicos, las artes y los deportes, y no sólo se centran en lo académico, aunque lo reconocen como elemento importante, de la misma forma que se plantea como objetivo dentro del proceso de Kumon: el papel de Kumon no es solamente enseñar matemáticas, es desarrollar todas esas capacidades que son enormes, y que van a ayudarnos no solamente en las matemáticas sino para muchas cosas en la vida, para desarrollar su personalidad, para fortalecer sus virtudes.

Para Díaz (2001, citado en Matijasevic et al., 2010), el papel de la pareja, los pares y los grupos a los que pertenezca el individuo 
son fundamentales, pues son un importante apoyo emocional. Este aspecto se destaca en las narrativas de los NNA; si estas relaciones están bien y equilibradas todo lo demás estará bien: [...] mantener todo balanceado o sea no puede uno pelearse todo el tiempo con la familia, [...] con los amigos no sería bonito pelear y en Kumon pues también porque somos amigos y es como el colegio. La escuela es un espacio de socialización en donde se da la adquisición de los aprendizajes de la sociedad, de su organización, de las objetivaciones del significado de la vida, y de su uso permitido por el lenguaje; de forma que marca las coordenadas de la vida en sociedad y la llena de objetos significativos, facilita la interacción con el mundo intersubjetivo que se comparte con los otros (la realidad propia y otras realidades de las que tiene conciencia).

Diferentes investigaciones muestran cómo aumenta el bienestar subjetivo con las relaciones de amistad, más allá de la cantidad de amigos, por ser relaciones de confianza y apoyo, es decir gratificantes (Moyano, 2007; citado en Matijasevic et al., 2010). Las relaciones son amistades, confío mucho entonces como que uno aquí consigue tener una buena calidad de vida. En sus propias palabras, este niño manifiesta que para tener una buena calidad de vida, necesita mantener buenas relaciones afectivas, dando importancia al poder confiar, y a su vez, lo liga como un elemento importante de la calidad del colegio en el que estudian, en relación no sólo a lo académico... en sus palabras: Un buen colegio [...] tiene que tener buenas bases académicas para entrar uno a la universidad [...] pero también tiene que tener bases sociales para que uno tenga unos valores claros. En sentirse bien es como encontrar un equilibro, entre más equilibrio más calidad para estudiar, para estar bien con sus amigos y todo en equilibro y planear las cosas para un futuro.

Asimismo, un clima agradable en el aula, basado en relaciones afectivas positivas, tanto entre los estudiantes como con el profesor, influirá positiva y directamente sobre el desarrollo infantil; también la equidad, el respeto a la libertad de iniciativa, la participación de los padres, la descentración y la autonomía (Beresaluce, 2009). Yo vengo todos los días (habla de Kumon) porque quiero, porque acá es bonito. Me gustaría que sean profesores buena gente, que no sean gritones, que enseñen bien, que los profesores sean confiables, que si alguien no entiende pues pueda preguntar. Estos elementos permitirán el desarrollo integral de los estudiantes, de los docentes, y en general, de toda la comunidad educativa, promoviendo el goce de un bienestar que propenda por una adecuada calidad de vida. No en vano, al preguntarles sobre las anécdotas que más recuerdan dentro de su proceso en Kumon, sus discursos se centran en las relaciones de amistad y noviazgo, que se convierten en referentes y complementos de los procesos académicos.

Comenius (s.f., citado en Martínez-Salanova) dedujo que el hombre debe convertirse en un todo armónico, por lo que deben desarrollarse plenamente todas sus potencialidades y habilidades, y no simplemente la razón. Los NNA reconocen que las relaciones que establecen en los contextos educativos no solamente satisfacen necesidades de amistad, sino que les permiten aprender de los otros, de sus experiencias, y siendo Kumon un espacio extra al colegio, amplía su círculo y les permite crear relaciones con personas mayores y menores, lo que favorece 
sus competencias comunicativas y de socialización: [...] empecé a hablarme contigo y a poder hablar con más gente, con Sofía y eso me ha parecido que ha sido una ganancia en Kumon, porque no solamente conozco gente de mi colegio sino que puedo conocer otro tipo de gente, y eso como que a uno le da más confianza, de pronto en un futuro en una universidad que no conozco a nadie, entonces ya con la experiencia de Kumon, de conocer más gente de otros lados, pues es mucho más chévere.

De otro lado, hay un interés por destacarse en los procesos académicos, con el fin de ser reconocido en un contexto que lo privilegia: Quiero acabar el G rápido (nivel de Kumon), por eso vengo todos los días, no quiero otra medalla de bronce, quiero la de plata. Es un reto personal ligado al premio público por su trabajo; esto igual es una familia y también es como en el colegio, cuando uno hace una feria en el colegio muestra obvio lo académico, no debería ser así, pero pues lo que más muestran es la vida social, hay logros que son producidos por lo académico [...] en el colegio siempre me ha ido muy bien, [...] peleaba con las profesoras porque ellas no sabían y yo sí. En el relato hace referencia a que no debería ser lo académico lo único que muestra, sin embargo pareciera que hay una adaptación a esta dinámica, por la que sí desea reconocimiento, es en lo académico en lo que debe sobresalir, aspecto que se relaciona con la discusión del siguiente apartado.

\section{La educación como expectativa potenciadora del proyecto de vida}

Esta subcategoría recoge los discursos de los NNA y los actores institucionales, a los que se hace referencia en cuanto a la educación como elemento que favorece la calidad de vida, al potenciar el proyecto de vida de los individuos. Lora, Chaparro y Rodríguez manifiestan que: "los individuos más educados tienen más opciones no sólo para satisfacer sus necesidades de consumo [...], sino también para sentirse autónomos, competentes y conectados" (Pombo, 2008, citado en Matijasevic et al., 2010), lo que coincide con la narrativa del asesor de Kumon, quien menciona que: la gente que estudia siempre está en la posibilidad de subir en la escala socioeconómica definitivamente. Para Sen (2000) esto serían las capacidades, descritas como la ventaja que tiene una persona para efectuar actos valiosos. En este sentido, la ventaja sería dada por la educación, y el resultado, los buenos modales y el ascenso económico.

Pérez, citando a Tonucci (2009), cree que el profesor no es el saber sino el mediador del saber. De esta manera, las orientadoras actúan como agentes potencializadores del desarrollo de esas capacidades, su tarea es descubrirlas y ayudarle al estudiante a desarrollarlas, facilitar su bienestar subjetivo, dentro del cual hay un componente cognitivo concebido como satisfacción con la vida, que puede abarcar desde la sensación de realización hasta la experiencia de fracaso o frustración (García 2002, citado en Matijasevic et al., 2010).

Nuevamente se destaca el papel del maestro, cuya labor va más allá de llenar de conocimientos a los estudiantes: que se usen esas capacidades desarrolladas, que estaban escondidas pero que ahora las sacaron a flote las orientadoras. Ese es el nuevo papel de la orientadora es descubrir [...] el potencial que son las capacidades latentes y hacer que se usen. Este discurso recuerda la forma en que Comenius (citado 
en Martínez-Salanova) definió la educación: "el arte de hacer germinar las semillas interiores que se desarrollan no por incubación sino cuando se estimulan con oportunas experiencias, suficientemente variadas y ricas y sentidas siempre como nuevas, incluso por quién la enseña".

Calvo (2008) afirma que el término informal en la educación pareciera remitir a un proceso caótico, carente de forma y asistemática, quizá esto ocurre porque estos procesos permiten flexibilidad que el sistema escolar no. En el caso de Kumon, los estudiantes manejan sus tiempos y horarios, y esto hace parte de la autonomía y autodisciplina que se busca formar: son ellos y ellas quienes trazan sus metas de avance a partir de su propio proyecto de vida, lo que les permite ver contenidos por encima de su grado escolar. El nivel más difícil fue el de las divisiones [...] ya estoy viendo temas que no he visto en el colegio, y me ha servido porque uno ya se sabe todos los trucos para lo que uno ve en el colegio. Su proceso en Kumon se vincula directamente con los académicos, y les brinda además la posibilidad de adquirir destrezas que complementan sus aprendizajes ${ }^{2}$, lo que para ellos se convierte en una ventaja: [...] una ventaja es que estoy viendo un nivel alto más alto que el de mi nivel escolar, eso aumenta mi calidad de vida hehe, pues porque a uno lo hace sentir bien y uno se siente bien siendo más que los otros, pues no menospreciándolos pero uno se siente bien que lo, no que lo alaben pero sí que le reconozcan el esfuerzo además de lo que uno hace en el colegio.

2 En el artículo El Método Kumon: Educación con calidad de vida (2011) publicado en El viaje y el viajero, a manera de metáforas de movilidades, desarrollo con más profundidad este aspecto.
Comenius (s.f., citado en Martínez-Salanova) decía que "a los hombres se les ha dado una oportunidad para la perfectibilidad continua e interminable, para la creatividad, para la educación permanente y para la autoeducación", la educación para estos NNA es un elemento que potencia su bienestar, y por ende su calidad de vida; no sólo potencia procesos como la autoestima sino que también hace que el aprendizaje adquiera un significado diferente: Lo de los niveles me ha parecido muy chévere porque he aprendido muchísimo, y ya me siento en el colegio que voy con una ventaja sobre los demás, que esa es la materia (matemáticas) a la que normalmente todo el mundo le tiene miedo, y es la que a mí más me gusta porque no tengo que estudiar, no tengo que hacer nada simplemente poner atención.

Si bien las narrativas de los NNA ponen a la educación como aquel camino que les implica sacrificios, y no siempre es lo que más disfrutan, la consideran necesaria para alcanzar las metas que se han trazado. Kumon es un valor agregado en su proceso educativo, que les permite adquirir confianza y ver contenidos por encima de los que ven en su grado escolar: cuando uno era chiquito pues le gustaba venir, a uno le parecía chévere, pero ya cuando es grande ya se aburre, pero uno piensa que eso va a ser bueno para la vida y que eso le va a servir en el colegio y todo. A pesar de que no es lo que más disfruta, lo reconoce como importante, logra trascender del deber al hacer. La educación tiene que ver con el poder ser y no con el deber ser, lo que significa que la educación es posibilidad, que se va construyendo y retroalimentando con el paso del tiempo, en un movimiento de espiral y no lineal. Se avanza, se retrocede, se comprende, se confunde. En resumen, la educación propende a la 
libertad a través de bifurcaciones interminables (Calvo, 2008).

En otro de los discursos manifiestan: me gustaría pasar más tiempo acá (en Kumon), sino que con el colegio no puedo porque tengo igual que sacar adelante otras materias, aquí aprendo más y puedo estar más tiempo con gente que no conozco y como por decirlo así volverme más culto. Mostrando que, contrario a lo que muchas veces se piensa, no hay un desinterés de los NNA por la educación; el ser humano es curioso por naturaleza y todo el tiempo desea aprender, pero sí hay un cuestionamiento de los estudiantes por los cómo, aspecto que se desarrollará mayormente en la siguiente subcategoría.

\section{Calidad de vida como la educación soñada}

En esta subcategoría se retomaron las narrativas de los NNA en las que hablan de cómo les gustaría que fuera la educación, y se pretende dar a conocer sus innovadoras ideas con los elementos que tendrían en cuenta, lo que para ellos debe ser lo más importante y qué debería tener un buen colegio.

Renzi y Tonucci (s.f) sostienen que los niños no son recipientes vacíos que hay que llenar de conocimientos; tienen sus propias vivencias y formas de pensar. Hay que escucharlos y crear entre todos el conocimiento; que son capaces de mantener su concentración durante un buen rato en un juego, pero que esto no parece interesar a la escuela porque no ayuda a elaborar lógicamente los datos. Afirman que en la escuela se debe tener más en cuenta el divertimento. Uno de los protagonistas de esta investigación propone: tener como dos horas de hacer lo que uno quiera o un hobbie. En los deportes pondría como todos los deportes, pero con tal de que cumplan en el colegio digamos haciendo tareas y que les vaya bien.

Para Tonucci:

La escuela debe ser un lugar bello, donde se pueda respirar cultura, haya música, arte, sea agradable y cómoda. Debe preocuparse por ofrecer a todo el mundo aquellas bases, aquellas motivaciones, aquellos modelos culturales imprescindibles para construirse en patrimonio de conocimiento, habilidades y competencias. (s.f.)

Este es un aspecto que los NNA entrevistados también destacan como parte importante del proceso educativo: académicamente tener bases en lo que yo pienso que es lo más importante que son las ciencias, la matemática, los idiomas y tener un lado musical o de artes para que los niños no solamente como que vean el lado aburrido... y hacer presentaciones cada año para que los niños pierdan ese miedo a enfrentarse a un multitud. Si bien en el sistema escolar ha sido tendencia ver la educación estética y física como elementos aislados y menos importantes dentro de los currículos, ahora muchas instituciones están incluyendo la educación corporal, como un eje transversal para el desarrollo integral de los estudiantes, lo que es reconocido y valorado por los NNA como un espacio de aprendizaje lúdico.

Otra de las propuestas apunta a la formación de hábitos desde la educación inicial, la que propone sea la principal: la parte más importante que debe ser el colegio es preescolar, porque si uno entra desde el primer curso ahí le enseñan muchas cosas que en el futuro le van a servir más, porque le van a enseñar los hábitos de cepillarse los dientes, pues a los de preescolar 
les tienen el cepillo. Si los NNA pasan la mayor parte del tiempo en el contexto escolar, éste debe entrelazarse con el trabajo que realizan los padres en el hogar y viceversa, así los niños y niñas encontrarán coherencia y desarrollarán autonomía, por ejemplo, en los procesos de autocuidado.

Con respecto a la estética en los contextos educativos, los NNA manifiestan la necesidad de espacios amplios, rodeados de naturaleza, aptos para la práctica de deportes y que favorezcan las estrategias educativas y el trabajo en el aula: Un buen colegio debe tener buenas instalaciones porque ahí va apoyando las estrategias y lo que uno usa. Otra narrativa dice al respecto: que tuviera un parquecito bonito para jugar y muchas florecitas y arbolitos. Beresaluce (2009) afirma:

La estructura física del aula un factor que influye de manera positiva, y tanto directa como indirectamente, sobre el desarrollo de las destrezas infantiles. Por un lado, la cantidad y calidad de los espacios, así como su adecuada disposición, permitirán a los niños permanecer concentrados, durante la realización de las actividades que les encomiende el profesor, al tiempo que establecen relaciones cordiales, incidiendo conjuntamente en la calidad del proceso educativo (efecto indirecto sobre el desarrollo). Por otro lado, un aula con una estructura física adecuada producirá un incremento en el desarrollo del niño (efecto directo). (p. 2)

Encaminado también al ambiente del aula, una de las niñas habla sobre el número de estudiantes por salón: que fueran poquitos niños en el salón, porque cuando son muchos pues no le ponen cuidado a todos. Este aspecto se relaciona con el deseo de reconocimiento por parte de los NNA que se desarrolló anteriormente, además el número de niños a los que tenga que atender el profesor, así como el número total de niños que se encuentran en el aula, facilitará, cuanto menor sea, la relación de actividades variadas, interesantes para todos y elegidas, en la medida de lo posible, por los niños. Esto beneficia, asimismo, al desarrollo infantil en todas las áreas (Beresaluce, 2009), a la vez que facilitará una relación más cercana entre el maestro y el estudiante, de tal forma que puede ayudarlo a potenciar sus habilidades a través del trabajo cooperativo.

Sobre las tareas para desarrollar en la casa, los NNA las consideran importantes y útiles para fortalecer sus aprendizajes, sin embargo en colegios privados como los de la capital colombiana, en donde los estudiantes salen de sus casas a las 5 o 6 de la mañana, y las jornadas se extienden hasta las 4 o 5 de la tarde, y que además el tráfico de la ciudad hace que el trayecto a la casa se prolongue por varias horas, muchos al llegar a las 6 de la tarde deben continuar con las labores, evitando que tengan la posibilidad de lograr otros aprendizajes, en espacios de conversación o juego con su familia y comunidad. Una de las niñas entrevistadas dice: pues no dejaría que dejaran tantas tareas porque uno llegar a la casa y tener que venir a Kumon, y aparte ir a la casa a hacer tareas pues es cansador entonces uno se acuesta muy tarde y al otro día tiene mucho sueño.

Renzi y Tonucci (s.f) hablan de la necesidad de dejar tiempo por las tardes a los niños, para que hagan cosas diferentes y luego tengan temas de conversación para hablar 
en clase. Esto requiere un mínimo de deberes escolares en casa, ya que pasan suficiente tiempo en el aula. Y agregan:

La escuela tiene que estar ajustada a sus necesidades, en la cual se haga más en menos tiempo. No puede ser que tras seis horas de clase los niños lleguen a casa con deberes. Los niños tienen que hacer cosas en casa, pero cosas que sepan hacer ellos solos. Si todos los niños hacen las mismas actividades y ven la misma tele luego en la escuela no tienen nada que contar a sus compañeros. (Renzi \& Tonucci, s.f.)

Otro elemento que se relaciona con lo estético, pero centrándose en lo individual y que es ampliamente criticado por los estudiantes es que los colegios creen normas respecto a la apariencia física: puede tener uno el pelo largo o cortico, verde, azul, amarillo eso no le va a quitar a uno las neuronas, mucha gente de esa que tiene el pelo largo que la discriminan socialmente es más inteligente que cualquier boba del colegio, pintarse las uñas, tener la falda alta, también adecuarse a lo que está ahora; otro de ellos menciona: no poner tantas reglas... digamos que no se puede usar manillas, yo creo que a veces en el colegio, digamos tener el pelo corto, yo creo que eso está mal porque uno va al colegio a estudiar no a fijarse en el pelo o en las manillas, uno va a estudiar y a aprender y en eso se verán los resultados.

Paradójicamente las escuelas buscan transmitir valores respecto a la igualdad y no discriminación, pero muchas veces sus prácticas llevan a los estudiantes a pensar que se contradicen y se guían por estereotipos culturales. Tonucci habla de las escuelas como espacios democráticos por lo que se debe dar más poder y libertad a los NNA, que éstos sean el centro de formación contando sus experiencias. En una entrevista el autor manifiesta:

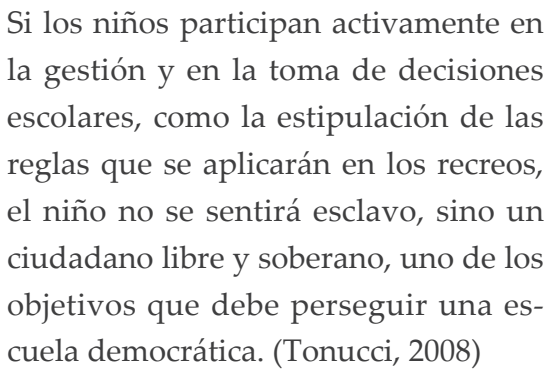

Si se hiciera de la escuela un espacio democrático y de construcción de normas conjuntas, muchos de los deberes pasarían de no tener sentido a ser respetados e incorporados, porque ellos mismos los propondrían y sabrían que objetivo se busca. Nuestro colegio sería como un club de amigos, manteniendo jerarquías obvio. Sería más como todos somos iguales [...] como una segunda casa [...] es que en algunas partes lo juzgan a uno [...] también tiene que tener unos parámetros teniendo en cuenta las cosas malas, porque digamos yo no dejaría fumar a los niños en el colegio pues porque fumar no está bien. Esto nos remite nuevamente a la propuesta con estructura democrática de la que habla Tonucci (2008):

Si contara con representantes de clase, un niño y una niña elegidos por sorteo, que se reúnen con el director de la escuela de vez en cuando, cada quince días, no para discutir de la ciudad, ni propuestas para el alcalde sino para discutir de la escuela, de cómo funciona, lo que les gusta, [...] Puede suceder que algunas de sus propuestas sean buenas y solucionen muchos problemas. 
Esto se observó a lo largo de este artículo, particularmente en este apartado, donde fueron muchas las propuestas hechas por los NNA, quienes mostraron interés por hacer parte de la construcción de los espacios educativos, principalmente la escuela, como un espacio propio y donde esperan sentirse a gusto.

\section{Narrativas de CAlidad DE VIDA DE NNA BOGOTANOS}

Luego del trabajo realizado con los NNA escolarizados y los actores institucionales de Kumon Instituto de Educación, acerca de las prácticas discursivas sobre calidad de vida, se observa que, si bien hay unos elementos objetivos que tienen que ver con indicadores de desarrollo para hablar de satisfacción de necesidades, es necesario involucrar elementos subjetivos, como ya se ha venido haciendo, que retomen elementos de los contextos sociales significativos de las personas; pero además es necesario que para la construcción de políticas públicas sobre calidad de vida, se ponga el tema en discusión con los directamente involucrados, y no sólo con las instituciones encargadas del desarrollo de los planes, pues son los primeros los que pueden proponer elementos que las instituciones no consideran, a la vez que se crea un sentido de pertenencia frente a los programas estatales, favoreciendo mejores resultados.

Si bien el objetivo era conocer las narrativas de los NNA y no las de actores institucionales, al involucrarlos se buscaba conocer los puntos de encuentro y desencuentro con los NNA sobre la calidad de vida, y a la vez retroalimentar particularmente las prácticas institucionales en el contexto de la investigación, de la Unidad Pablo VI de Kumon Instituto de Educación. De esta manera, dentro de la categoría relación calidad de vida y educación, se encontraron principalmente cuatro grandes prácticas discursivas: 1) calidad de vida como deseo y expectativa de afecto de los maestros hacia los NNA; 2) calidad de vida como educación integral: relaciones de respeto, reconocimiento y convivencia entre pares y con maestros; 3) la educación como expectativa potenciadora del proyecto de vida, y 4) calidad de vida como la educación soñada.

Se retomaron principalmente las narrativas de los actores institucionales, en las que relacionan la calidad de vida con su labor como educadores, poniendo como fin y meta del proceso de sus estudiantes la felicidad y el bienestar, y como medio los procesos educativos, involucrando en ellos no sólo lo académico, sino también los procesos afectivos y relacionales como favorecedores del aprendizaje. Se resalta el establecimiento de relaciones cercanas con los estudiantes, lo que permite conocer sus habilidades, capacidades y oportunidades, las cuales pueden ser utilizadas favorablemente en el trabajo que se desarrolla con ellos en Kumon. En este punto se evidencia, por parte de los NNA, una tensión en la escuela que centra la educación en la transmisión de conocimiento, frente a una escuela que, ellos desean, incluya en el proceso educativo las relaciones afectivas y el respeto entre pares, maestros y estudiantes.

El involucramiento del componente afectivo, como eje transversal en la educación, se ha convertido no sólo en una responsabilidad y una necesidad de la escuela, 
sino también en una solicitud de los NNA (e incluso de los mismos docentes), quienes pasan la mayor parte del tiempo en el colegio, lo que hace que la estabilidad y el bienestar en las relaciones que crean con pares y maestros, se conviertan en un factor importante para hablar de su calidad de vida. Los NNA refieren la necesidad de reconocimiento por parte de los actores institucionales y, en el caso de nuestros protagonistas, centran sus recursos para lograr destacarse en el ámbito académico, componente que en sus contextos sociales significativos tiene un alto valor como potenciador de sus proyectos de vida. La educación, y específicamente, el acceder a Kumon, son recursos que favorecen el desarrollo de capacidades, que, a futuro, esperan poner en marcha para el logro de sus metas profesionales y personales.

Finalmente, proponen que la educación les brinde espacios de participación y opinión, donde sus propuestas sean tenidas en cuenta, pues si bien reconocen que el componente académico es primordial, éste debe ser ampliado en pro de una educación integral que, como se mencionó, involucre elementos afectivos, además del arte, los idiomas y los deportes como espacios lúdico recreativos, que permitan aprendizajes útiles en el mundo actual y respondan a su expectativas. De igual forma, desean ser partícipes de la toma de decisiones y la construcción de normas; no desean una escuela laxa, puesto que reconocen la importancia de reglas claras, pero sí proponen la existencia de reglas con propósitos claros que ellos conozcan, de tal forma que no será un deber respetarlas sino parte de la cultura de la escuela, además de contribuir a sus procesos educativos éticos.

\section{REFERENCIAS}

Arenas, A. (6 de octubre de 2008). Francesco Tonucci: "Cada momento educativo se debería empezar oyendo a los alumnos" (Entrevista). Ideal en clase.

Arenas, K. Jaramillo, J. \& Riaño, P. (2004). Descripción y comprensión contextual de la pauta relacional y las narrativas del juego patológico en los contextos sociales significativos del jugador (Tesis de pregrado no publicada). Universidad Santo Tomás, Bogotá, Colombia.

Aristóteles (1873/2005). Moral a Nicómaco. (Azcarate, P. Trad.). Madrid: Biblioteca de Instrucción y Recreo.

Banco Interamericano de Desarrollo. (2008) Calidad de vida. Más allá de los Hechos (E. Lora, Ed.). Ciudad de México: Fondo de cultura económica.

Beresaluce, R. (2009). Calidad de vida en la escuela. La calidad cono reto en las escuelas de educación infantil al inicio del siglo XXI. Las escuelas de Reggio Emilia, de Loris Malaguzzi, como modelo a seguir en la práctica educativa (Tesis doctoral no publicada). Universidad de Alicante, España.

Beuchot, M. (2002). Perfiles esenciales de la hermenéutica. Ciudad de México: Universidad Nacional Autónoma de México.

Bonilla, E., \& Rodríguez P. (1997). Más allá del dilema de los métodos. Bogotá: Ediciones Uniandes. ( $\left.2^{\mathrm{a}} \mathrm{ed}\right)$.

Binimelis, A., \& Canales, M. (1994). El Grupo de Discusión. Revista de Sociología, 9, 107-116.

Bruner, J (1991). Actos de significado, más allá de la revolución cognitiva. Madrid: Alianza.

Bruner, J. (1997). La educación puerta de la cultura. Madrid: Visor. 
Buitrago, J., Cardona, J., Gómez, M., Páez, D., Riaño, P., \& Salazar, M. (2011). Prácticas discursivas sobre calidad de vida de niños, niñas y jóvenes en contextos multidiversos frente a la política pública: Armenia, Pereira, Santuario (Risaralda), Manizales y Bogotá. En L. Amador, \& M. Salazar (Eds.), El viaje y el viajero, a manera de metáforas de movilidad-es. Manizales: Red Suma.

Calvo, C. (2008). Del mapa escolar al territorio educativo. Santiago: Nueva Mirada.

Camps, V. (2002) Educación y calidad de vida. En Ayuntamiento de Gijón (Ed). Proyecto Educativo de Ciudad. Gijón: Ayuntamiento de Gijón.

Casa Editorial El Tiempo, Fundación Corona y Cámara de Comercio de Bogotá. (2009, Abril). Evaluación de los cambios en la calidad de vida en Bogotá durante 2008. Sector Educación. Recuperado el 16 de febrero de 2010, de www.bogotacomovamos.org

Casa Editorial El Tiempo, Fundación Corona y Cámara de Comercio de Bogotá. (2011). Encuesta de percepción ciudadana 2011. Recuperado el 23 de septiembre de 2011 de www.bogotacomovamos.org

Castillo, J., Castillo, C., \& Esguerra, G. (2011). Conceptualización y medición de la calidad de vida en la infancia. Diversitas: Perspectivas en Psicología, 7 (1), 103 - 124.

Concejo de Bogotá. (2008). Plan de desarrollo "Bogotá positiva: para vivir mejor". ACUERDO No. 308 DE 2008. Recuperado en marzo de 2010 de www.bogota.gov.co

Congreso de la República de Colombia. (2006). Código de la Infancia y la Adolescencia. Ley 1098 de 2006. Bogotá: Congreso de la República de Colombia.
Contraloría de Bogotá. (2010). Informe de auditoría gubernamental con enfoque integral. Recuperado el 26 de enero de 2011 de: www.bogota.gov.co

Departamento Nacional de Planeación. (2010). Bases del Plan Nacional de Desarrollo 20102014: Prosperidad para todos. Recuperado en enero de 2011 de: www.dnp.gov.co

Epston, D., \& White, M. (1993). Medios narrativos para fines terapéuticos. Madrid: Paidós.

Hospital Pablo VI Bosa. (s.f.) ¿Qué es un grupo focal? Recuperado el 10 de mayo de 2009 de: www.hospitalpablovibosa. gov.co

Martínez-Salanova, E. (s.f) Comenius. Recuperado el 20 de noviembre de 2011 de http:// www.uhu.es/cine.educacion/figuraspedagogia/0_comenius.htm\#La_propuesta_pedag \%C3 \%B3gica_de_Comenius

Matijasevic, M, Ramírez, M. \& Villada, C. (2010). Bienestar subjetivo: Una revisión crítica de sus resultados, alcances y limitaciones. RegionEs, 5(1), pp. 5- 39.

Pérez, A. (2009). La evaluación en el CBC: un desafío de la enseñanza. Jornada de intercambio de experiencias pedagógicas, Buenos Aires, Argentina.

Pourtois. J. \& Demet. H. (1992). Epistemología e instrumentación en las ciencias humanas. Madrid: Herder.

Prisco, A., Renzi, D. \& Tonucci, F. (s.f). Proyecto internacional "La ciudad de los niños del "del Instituto de Ciencias y Tecnologías del Conocimiento de Roma. Recuperado el 23 de noviembre de 2011 de www.lacittadeibambini.org

Programa de las Naciones Unidas para el Desarrollo - PNUD. (2008, Julio). Bogotá una apuesta por Colombia. Informe de desarrollo humano. Recuperado el 10 de abril de 2010 de www.pnud.org.co 
Salazar, M. (2010). Calidad de vida y niñez: una aproximación comprensiva a la política social desde los derechos en el municipio de Manizales 2003-2010 (Disertación doctoral no publicada). Cinde y Universidad de Manizales, Manizales, Colombia.

Sen, A. (2000). Desarrollo y libertad. Barcelona, España: Planeta.
Tonon, G. (2005). Apreciaciones teóricas del estudio de la calidad de vida en Argentina. Hologramática, 2 (1), 27- 49. Valles. M. (1999). Técnica cualitativa de investigación social: Reflexión metodológica y práctica profesional. Síntesis Sociología. 